\title{
Blocking in human place learning: Evidence from virtual navigation
}

\author{
DEREK A. HAMILTON and ROBERT J. SUTHERLAND \\ University of New Mexico, Albuquerque, New Mexico
}

\begin{abstract}
The associative conditioning phenomenon of blocking has recently been demonstrated in nonhuman place-learning paradigms, including the Morris water task (MWT). We demonstrate blocking in humans learning to place navigate in a computerized version of the MWT. Participants trained to locate an invisible goal with an initial set of distal cues were deficient in learning to locate the goal with a set of cues inserted later during training. This outcome is inconsistent with the spontaneous integration of novel environmental features into a unified spatial representation proposed by cognitive mapping theory (O'Keefe \& Nadel, 1978) and suggests that error-correcting associative learning principles operate in the human spatial mapping system.
\end{abstract}

In this report, we draw upon evidence from human performance in a computerized version of the Morris water task (the virtual Morris water task; VMWT) to contrast basic associative and cognitive mapping (O'Keefe $\&$ Nadel, 1978) accounts of place learning. The Morris water task (MWT; Morris, 1981) has been employed extensively to measure place learning in rats and has been useful in identifying the neurobiological underpinnings of place learning from systems to molecular levels of description. In the MWT, animals learn to locate a hidden escape platform in a circular pool of opaque water. Rats are capable of swimming directly to the goal location by using only a few distal cues (Morris, 1981; Rodrigo, Chamizo, McLaren, \& Mackintosh, 1997), and the removal of cues from the environment leaves spatial localization intact provided that any two cues remain (Prados $\&$ Trobalon, 1998). Thus, performance relies on a representation of the configuration of environmental features in which no single feature is necessary or sufficient to guide navigation. It is now well established that performance in the hidden platform version of the MWT depends on the integrity of the hippocampal formation (the hippocampus, the dentate gyrus, and the subicular cortex; Morris, Garrud, Rawlins, \& O'Keefe, 1982; Sutherland, Whishaw, \& Kolb, 1983), and initial acquisition is related to hippocampal synaptic plasticity (Morris \& Frey, 1997; Moser, Krobert, Moser, \& Morris, 1998). Measures of human performance in the VMWT indicate that normal, college-aged individuals locate the hidden goal by using virtual distal cues in a manner similar to that of rats (Astur, Ortiz, \& Sutherland, 1998). A further similarity between MWT and VMWT learning has been demonstrated recently in the form of hippocampal in-

The authors thank Frank Logan and two anonymous reviewers for their helpful comments on an earlier version of this article. Correspondence concerning this article should be addressed to D. A. Hamilton, Logan Hall, Department of Psychology, University of New Mexico, Albuquerque, NM 87131 (e-mail: dahamilt@unm.edu). volvement in VMWT acquisition and performance. $\mathrm{Pa}$ tients with unilateral mesial temporal lobe damage that includes the hippocampus fail to solve the task, and in a preliminary evaluation, bilateral hippocampal activation in normal individuals has been observed with functional magnetic resonance imaging (Astur, Brown, Price, \& Sutherland, 1998). In addition to the hippocampal formation, a widely distributed set of neural structures has been linked to spatial learning in nonhuman animals. These include the entorhinal cortex (Schenk \& Morris, 1985), the medial septum (Kelsey \& Landry, 1988; Sutherland \& Rodriguez, 1989), the nucleus accumbens (Sutherland \& Rodriguez, 1989), mammillary bodies (Sutherland \& Rodriguez, 1989), the anterior thalamus (Sutherland \& Rodriguez, 1989), the medial frontal cortex (Sutherland, Kolb, \& Whishaw, 1982), the posterior parietal cortex (Kolb, Sutherland, \& Whishaw, 1983), the retrosplenial cortex (Sutherland, Whishaw, \& Kolb, 1988), the medial caudate-putamen (Whishaw, Mittleman, Bunch, \& Dunnett, 1987), and the perirhinal cortex (Wiig \& Bilkey, 1994a; but see Wiig \& Bilkey, 1994b, and Glenn \& Mumby, 1998). Thus, even though the hippocampal formation plays a central role in place navigation, the observed properties of place learning emerge through the interaction of processes taking place in a widely distributed set of neural circuitry.

An unresolved issue concerns the principles by which acquisition of the spatial information supporting navigation proceeds. Does place learning rely on the same associative principles as Pavlovian or operant conditioning, or does it represent a departure for which additional or unique principles are required (Gallistel, 1990; Nadel, 1991; Nadel \& Willner, 1980; Tolman, 1948)? According to O'Keefe and Nadel's (1978) cognitive mapping theory, the latter is true. Under this view, place learning is governed by a specialized system localized in the hippocampus that creates and maintains a unified map-like representation of the environment in which the spatial relationships among the features of the environment are pre- 
served. Thus, the cognitive map contains data from which new information, such as novel trajectories to a goal location, could be calculated. Whenever there is a mismatch between the current perception of the environment and the stored representation, the organism explores the environment and integrates new features into and deletes removed features from the map. This updating operation occurs automatically, in an all-or-none manner and independently of goal-oriented behavior (see O'Keefe \& Nadel, p. 95); hence, once the map has been created, updating proceeds more rapidly than would building a new map. In this way, acquisition of the information supporting place navigation follows distinctly different processes than those postulated to operate by current theories of associative learning.

Associative accounts of place learning draw upon a rich history of research and theory in nonspatial learning domains in which learning is described in terms of the associative strength among stimuli and events and the error-correcting rules by which modifications of associative strengths occur (Mackintosh, 1975; Pearce \& Hall, 1980; Rescorla \& Wagner, 1972). Associative strengths are modified when expectations regarding the predicted event(s) differ from what is actually experienced, with the goal being to reduce the error in prediction. If similar associative learning principles (Biegler \& Morris, 1999; Mackintosh, 1997; Rodrigo et al., 1997; Rudy \& Sutherland, 1995; Sutherland \& Rudy, 1989) operate in spatial learning, phenomena routinely observed in Pavlovian and operant conditioning paradigms should exhibit in place learning (Morris, 1981). One such phenomenon is blocking, which has been observed in human (Hinchy, Lovibond, \& Terhorst, 1995; I. Martin \& Levey, 1991) and nonhuman (Kamin, 1969) Pavlovian conditioning. Blocking occurs when a previously established predictive relationship between a conditioned stimulus (CS) and an unconditioned stimulus (US) disrupts or prevents learning an equally predictive CS as a signal for the same US. In the spatial domain, this would be analogous to learning to use several cues to guide navigation to a goal and then having new, redundant cues added. Under an error-correcting associative account, the initial cues should block use of the added cues to the degree that the initial cues already provide good predictors of the goal location. That is, the new cues provide no new information that can improve prediction of the goal location. Conversely, cognitive mapping principles suggest that the added cues should be learned as well as the initial cues, as a result of novelty-driven exploration of the environment and subsequent automatic updating of the cognitive map.

Alternatively, place learning could be fundamentally associative in nature, yet could follow different rules of acquisition than the error-correcting principles of the associative learning theories described above. For example, Hebb (1949) suggested that the neural mechanism of associative learning involves the strengthening of neural connections on the basis of contiguous activity. When a pre- and a postsynaptic cell are coactive, their connection strength is increased, making the presynaptic cell more efficient at inducing activity in the postsynaptic cell. Such a learning algorithm is sensitive to correlations between stimuli and events without respect to previously established relationships (McNaughton \& Morris, 1987). Alterations following long-term potentiation (LTP) that affect the efficacy of synaptic transmission may represent the physiological basis of Hebb's postulate. Because LTP has been demonstrated and extensively characterized within pathways of hippocampal circuitry, it has been suggested that Hebbian synaptic modification principles may operate in spatial learning (Morris \& Frey, 1997; see, also, Burgess \& O'Keefe, 1996, and McNaughton \& Morris, 1987). Because Hebbian learning is independent of the goal-predictiveness of stimuli already available in the environment, blocking should not occur in the spatial domain.

A small but consistent body of nonhuman animal work has demonstrated blocking in the MWT (Rodrigo et al., 1997; Weisend et al., 1995) and in similar place-learning paradigms (Biegler \& Morris, 1999). Collectively, these studies have shown that prior learning of goal-predictive cues disrupts acquisition of equally predictive cues inserted later in training. We tested the generality of these findings, using a standard blocking procedure in the VMWT (see Table 1). A demonstration of blocking would suggest the operation of error-correcting associative learning in human place learning and would be inconsistent with Hebbian associative learning principles and with current statements of cognitive mapping theory.

\section{METHOD}

\section{Participants}

The participants were 25 male and 41 female University of New Mexico undergraduate students ranging in age from 18 to 54 years $(M=22.5, S D=7.10)$ who participated as part of a course requirement. Participation was restricted to individuals with normal or corrected vision and with no prior experience with this or other virtual place-learning experiments. None of the participants reported having a history of neurological problems. Assignment to conditions was random.

\section{Materials}

The three-dimensional computerized environment consisted of a circular pool located in the center of a room with a square floor plan (see Figure 1). The four distal room walls were visually identical and had an equal height:length ratio (approximately 1:10). An opaque blue water pattern was used to create the pool's surface. A dark random dot pattern was repeated around the circumference of the pool to form the pool wall, which extended above the water's surface by approximately $15 \%$ of the distal wall height. A square platform covered $1.75 \%$ of the area of the pool surface and, when

Table 1

Cue-Set Assignments for Each Phase of the Experiment

$\begin{array}{lccc}\text { Group } & \text { Phase 1 } & \text { Phase 2 } & \text { Phase 3 } \\ \text { Training } & \text { Training } & \text { Probe trial } \\ \text { Blocking } & \text { cue set A } & \text { cue sets A + B } & \text { cue set B } \\ \text { Control I } & - & \text { cue sets A + B } & \text { cue set B } \\ \text { Control II } & \text { cue set C } & \text { cue sets A + B } & \text { cue set B }\end{array}$




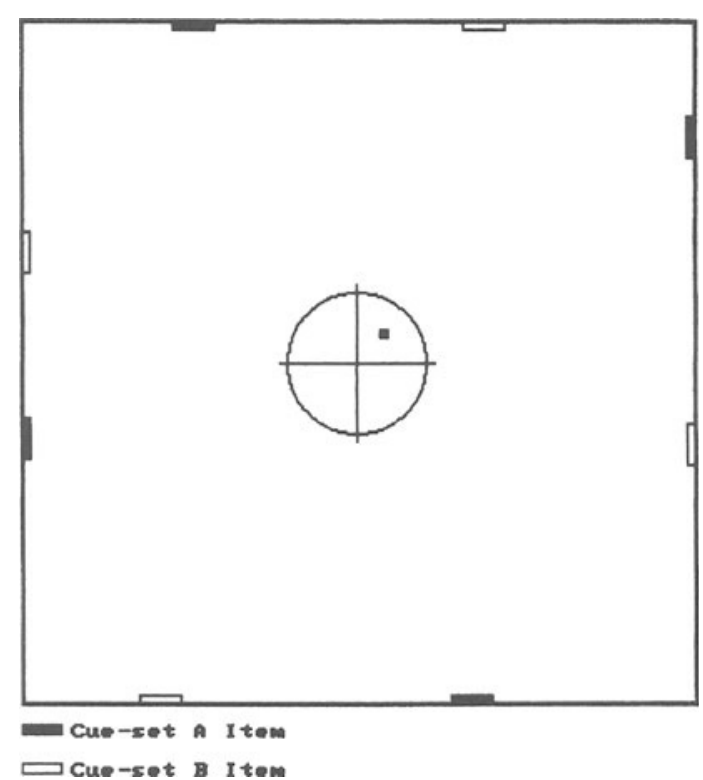

Figure 1. Layout of the virtual environment, including the pool, platform, distal walls, and cues in Phase 2. The four points at which quadrant demarcations meet the perimeter of the pool were used as starting locations. Note: The layout is to scale; however, the distal cues did not extend out from the distal room walls.

visible, extended vertically halfway to the top of the pool wall. The location of the platform was fixed throughout the experiment in the center of one quadrant. Three sets of four cues were created to serve as additional distal environmental features. All the environmental features were easily distinguishable from the rest of the environment; however, only the distal cues unambiguously predicted the platform location. All the cues were square, of equal area, and covered roughly $5 \%$ of a single distal wall. Distal cue distances from the pool wall varied from 2 to 2.25 times the diameter of the pool. Individual cues from the same cue set could be not placed in adjacent distal wall octants, which were determined by dividing each of the four distal walls vertically into equal halves (see Figure 2). The cues were placed flush with the distal wall, and all the cues were placed off center vertically and horizontally within their assigned octant by a random amount. Placement of individual cues was also constrained in such a way that no single cue provided a marker for direct navigation to the platform location from any of the four starting locations. Auditory feedback consisted of a bell, an aversive, discordant sound, and the sound of moving water, which accompanied forward movement through the pool.

A computer controlled the presentation of the environment, auditory feedback, and data collection. Visual aspects of the experiment were displayed on a 17 -in. color monitor, and auditory feedback was delivered via amplified speakers external to the computer. The up (forward), left, and right arrow keyboard keys were used for navigation; backward navigation was not possible. Traversal of a virtual distance equal to the diameter of the pool took a minimum of approximately $4 \mathrm{sec}$ to complete, and a full rotation in the absence of forward movement required approximately $2.5 \mathrm{sec}$.

\section{Design and Procedure}

The experiment was conducted in three phases (see Table 1). Trial blocks consisted of one trial for each of four starting points (see Figure 1). The order of starting locations was randomized within trial blocks. The participants were randomly assigned to one of three groups: the blocking group or one of two control groups (I or II). During Phase 1, the blocking group completed five hidden platform trial blocks with cue set $\mathrm{A}$ in the environment. Control Group I completed no trials during Phase 1, and Control Group II
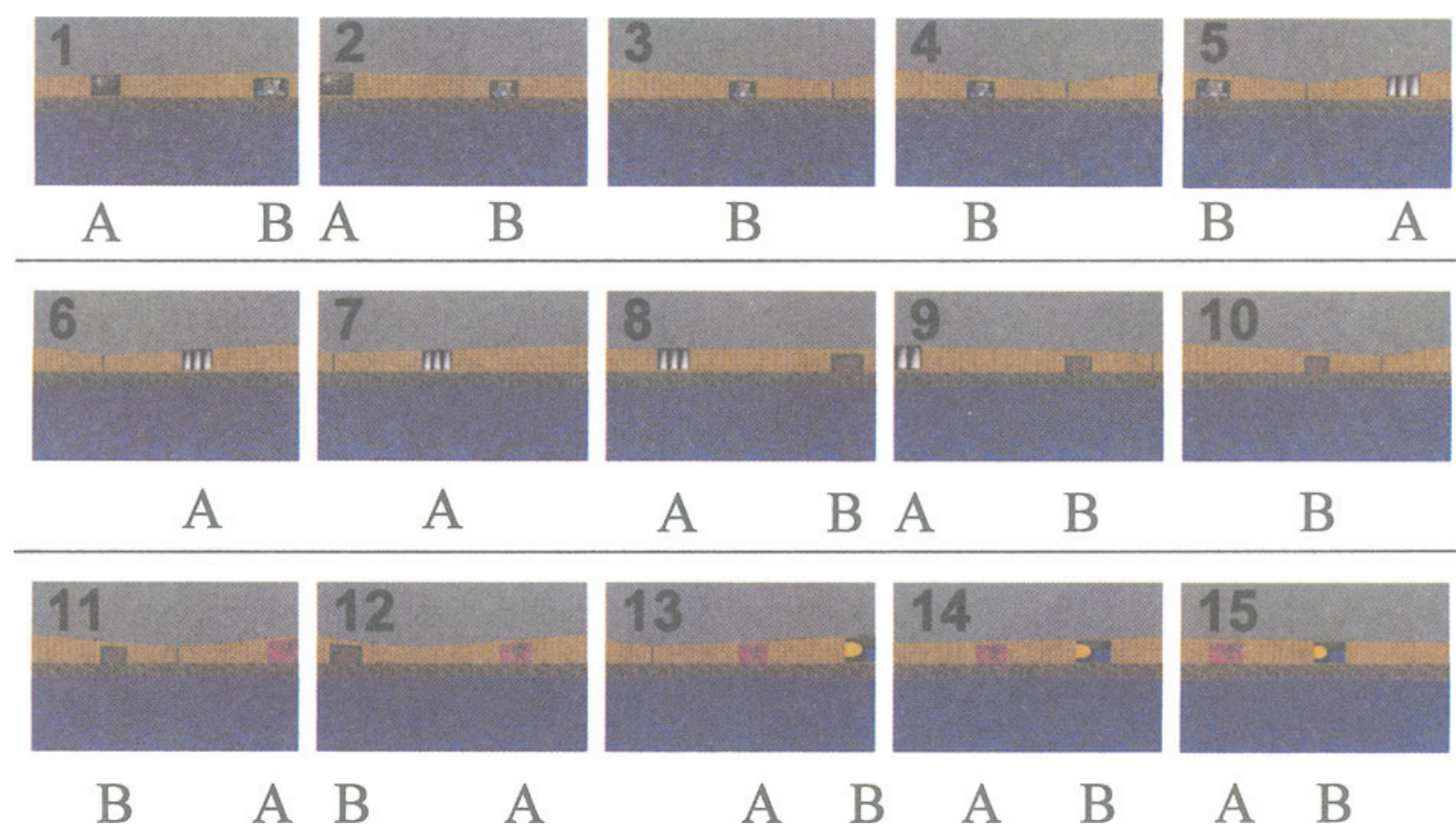

Figure 2. Views of the virtual environment during Phase 2, taken from the center of the pool. Each successive view represents a $12^{\circ}$ rotation to the right from the previous view. Elements from cue set $A$ and cue set $B$ are labeled below each image. 
completed five hidden platform trial blocks with cue set $\mathrm{C}$ in the environment. Control Group II was included to control for the amount of training in the virtual environment and for acquisition of nonspecific navigational skills. In Phase 2 , each group completed three hidden platform trial blocks with cue sets $A$ and $B$ in the environment. Pilot work revealed that three blocks of training were sufficient for the participants to reach asymptotic performance on all measures. Phase 3 consisted of a single probe trial in which the platform and cue set A were removed from the environment.

The participants were told that they would begin each trial facing the perimeter wall of the virtual pool and that their goal was to escape from the water by finding the submerged platform as quickly as possible. No information regarding useful strategies, the constancy of the platform location, the goal-predictiveness of environmental cues, or the variability and number of starting locations was provided to the participant. A limit of $60 \mathrm{sec}$ was allotted to locate the goal, after which the platform was made visible and the participants were prompted with a visually presented verbal message and aversive auditory feedback to swim to the platform. Auditory feedback was provided when the platform was found, and a verbal message was displayed stating this fact. Starting locations for Phase 3 probe trials were selected at random from the two locations furthest from the platform location. No-platform probe trials were $30 \mathrm{sec}$ in length, after which the environment was removed from the display. The task took between 20 and $40 \mathrm{~min}$ to complete.

\section{RESULTS}

Prior to data analysis, we classified participants as place learners or indirect on the basis of performance during the final block of Phase 2 training. This was undertaken to identify participants who, during training, acquired and used the spatial relationships among the goal and the distal cues to guide navigation. Two observers without knowledge of group membership performed this classifi- cation (see Figure 3 for examples of indirect and place learner swim paths). Those participants consistently taking direct paths to the goal location were classified as place learners. The remaining participants were classified as indirect, either because they adopted circuitous strategies, such as looping around the pool until locating the goal, or because they consistently moved a certain distance from the start location in a given direction in an attempt to locate the goal. Of the 66 subjects, 41 were classified as place learners and 25 as indirect. The expected frequency of participants classified as place learners was not confounded with group membership $\left[\chi^{2}(2\right.$, $N=41)=1.37, p=.51]$, nor were there unexpected differences in the proportion of males and females classified as place learners within each group [blocking: $\chi^{2}(1, N=$ 13) $=0.08, p=.78$; Control I: $\chi^{2}(1, N=11)=2.27, p=$ .13; Control II: $\left.\chi^{2}(1, N=17)=0.06, p=.81\right]$.

We report analyses of five performance measures: latency to locate the platform, latency to reach the pool quadrant containing the platform, heading error (the deviation from a direct path to the goal when the participants had traveled a distance equal to $50 \%$ of the pool radius from the start location), the swim path length from the starting point to the goal quadrant, and the percentage of total swim path length traveled within a region surrounding the platform comprising $50 \%$ of the pool area. Comparison of mean place learner and indirect performance measures during the final training block yielded significant differences in latency to locate the goal $[t(64)=3.38]$, latency to reach the goal quadrant $[t(64)=$ $3.45]$, heading error $[t(64)=3.95]$, swim path length before reaching the goal quadrant $[t(64)=4.22]$, and
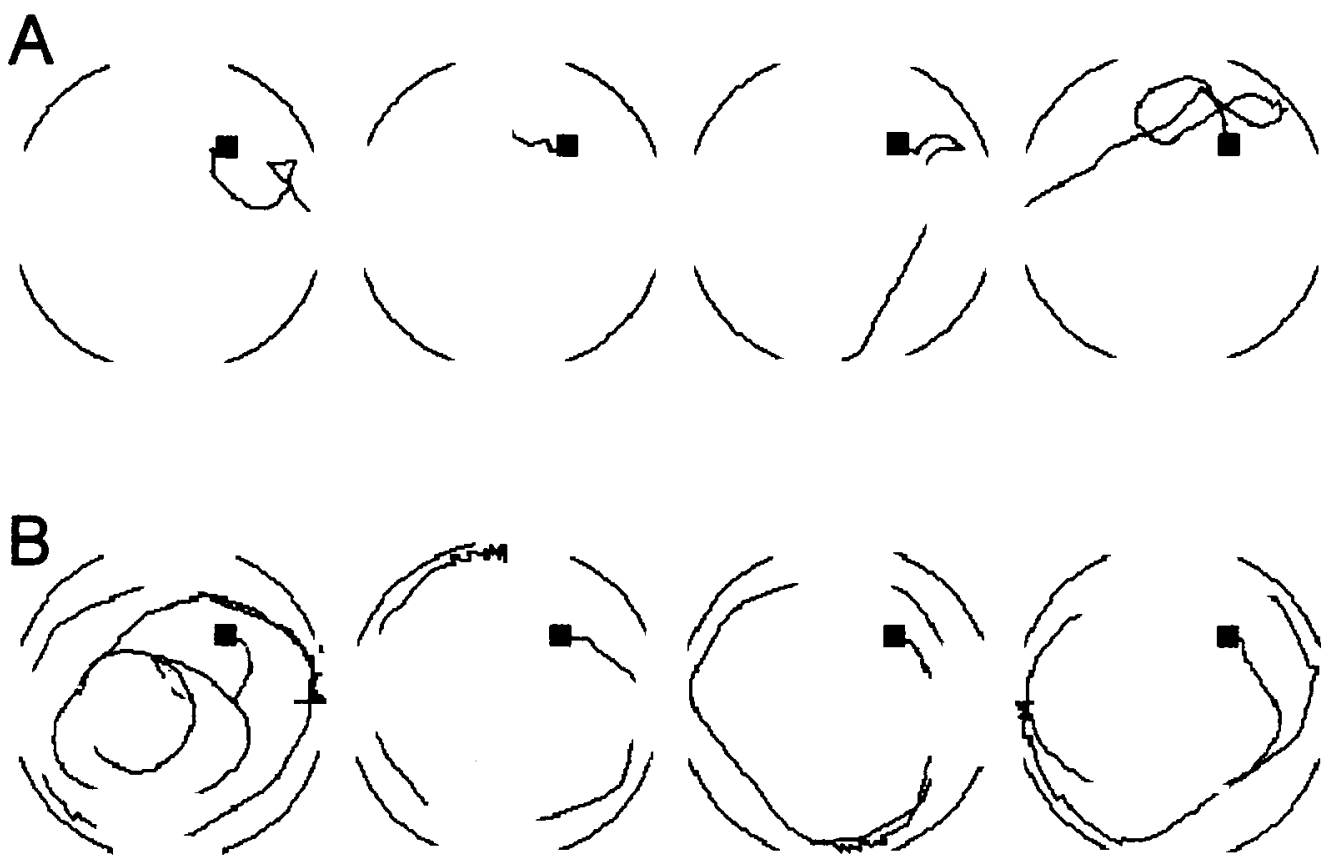

Figure 3. Examples of place-learner (A) and indirect (B) swim paths during the final block of Phase 2 training. 
percentage of total swim path length in goal region $[t(64)=3.94$, all $p s<.05]$. All effects were in the direction of better performance by place learners. For all subsequent analyses, we report an analysis with all the participants (place learner and indirect) included and an analysis limited to place learners.
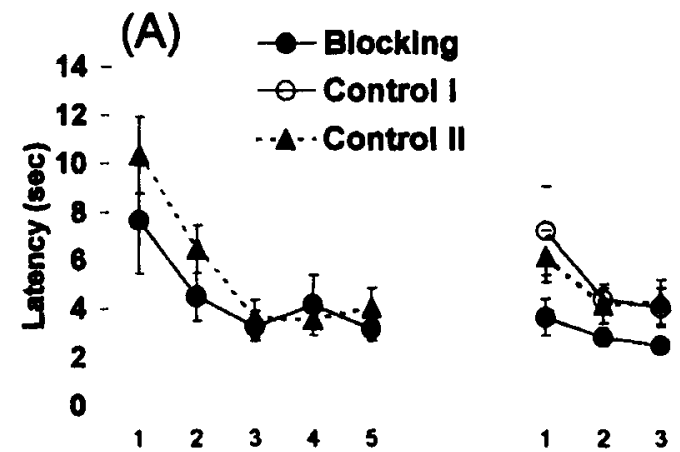

Phase 1

Phase 2

(blocks of 4 trials)<smiles>[CaH]</smiles>

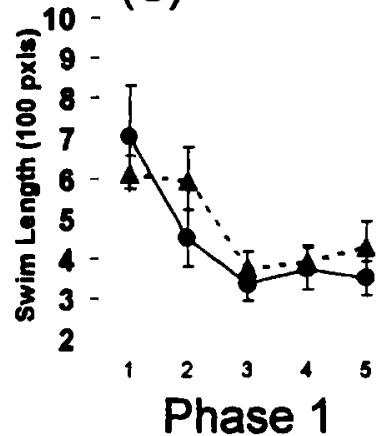

(blocks of 4 trials)
Mean performance measures for the four final training block trials (Block 3 of Phase 2) were calculated for each participant (see Figure 4 for a graph of mean performance measures during Phase 1 and Phase 2 training). There were no significant group differences on any performance measure when place learner and indirect

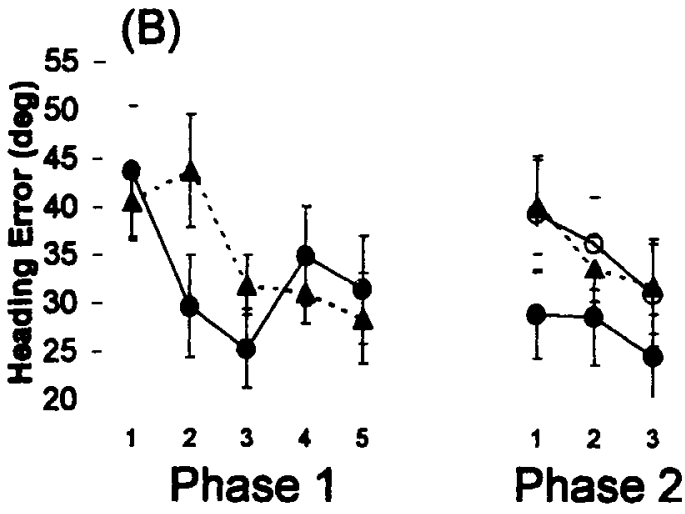

(blocks of 4 trials)

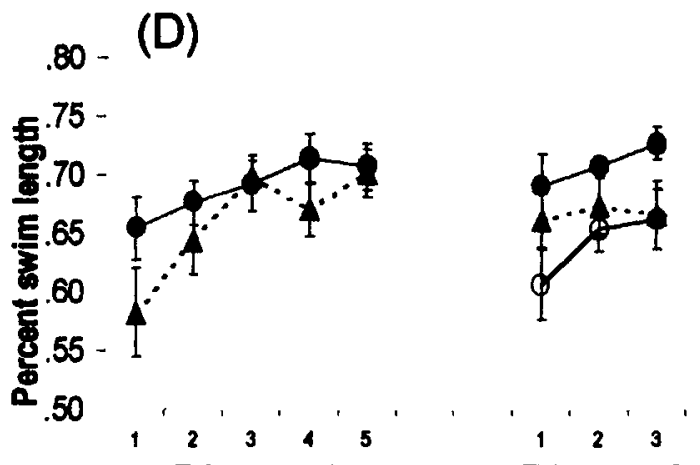

Phase 1

Phase 2

(blocks of 4 trials)
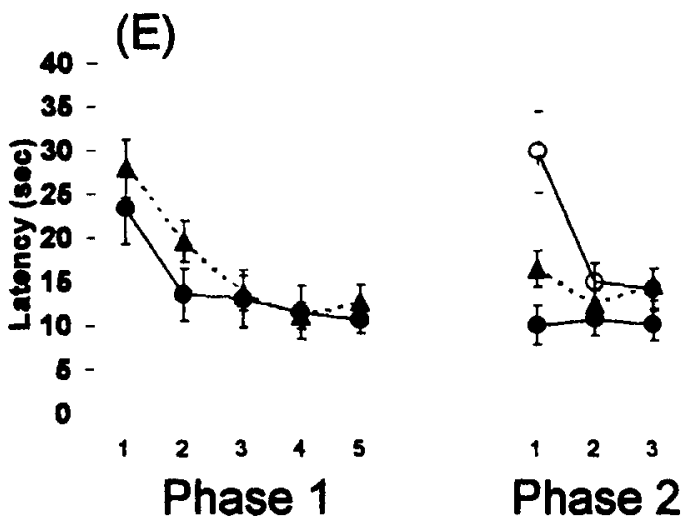

(blocks of 4 trials)

Figure 4. Phase 1 and Phase 2 training measures. (A) Mean latency $\pm 1 S E M$ to reach the goal quadrant. (B) Mean heading error $\pm 1 S E M$ calculated when a distance equal to $25 \%$ of the pool radius had been traveled from the start location. (C) Mean swim length \pm 1 SEM from start location to the goal quadrant. (D) Mean percentage of total swim length $\pm 1 S E M$ in the goal region (a circular region centered on the platform that comprised $50 \%$ of the total pool area). (E) Mean latency $\pm 1 S E M$ to locate the hidden platform. Note: Means and $S E M$ s plotted here were calculated only for place learners. 
participants were included in the analysis or when the analysis was limited to place learners (all $p s>.10$ ). Phase 3 probe trial performance measures were subjected to an analysis of covariance (ANCOVA), with mean performance during the final block of training as a single covariate (see Figure 5). For ANCOVAs including all the participants, there was a significant group effect in swim path length to reach the goal quadrant $[F(2,62)=3.37$, $p<.05]$. The blocking group swam significantly further than both control groups before reaching the goal quad$\operatorname{rant}[t(62)=2.22, p<.03]$. No differences between control groups in swim length to the correct quadrant were obtained $[t(62)=1.30, p=.197]$. Group differences in heading error approached significance $[F(2,62)=3.00$, $p=.057]$ and showed a trend in the direction of greater heading error for the blocking group as compared with controls. No further ANCOVAs including all the participants in the analysis revealed significant group differences (all $p$ s $>.22$ ).

ANCOVAs limited to place learners yielded significant group differences in latency to reach the goal quadrant $[F(2,37)=3.50]$, percentage of swim path length in the goal region $[F(2,37)=3.34]$, swim path length before reaching the goal quadrant $[F(2,37)=6.36]$, and heading error $[F(2,37)=4.64$, all $p$ s $<.05]$. The blocking group performed significantly worse, when contrasted with both control groups, on each measure: latency to reach the goal quadrant $[t(38)=2.50]$, percentage of swim path length in goal region $[t(38)=2.53]$, swim path length before reaching the goal quadrant $[t(38)=3.48]$, and heading error $[t(38)=2.95$, all $p \mathrm{~s}<.02$; Figure 6 shows examples of blocking and control group swim paths during the probe trial]. No significant performance differences between the control groups were obtained (all $p s>.37$ ].

Finally, a paired $t$ test comparing blocking group performances for the final block of Phase 1 and the initial block of Phase 2 was performed. No differences were obtained whether the analysis included all the participants or was limited to place learners (both $p s>.20$ ). Note that no such comparison can be performed for Control Group I, and the cue sets in Control Group II's environment were completely changed, so changes in performance would not be unexpected.

\section{DISCUSSION}

We interpret these results as being a clear demonstration of blocking in human place learning. The presence of distal environmental features that support spatial localization interfered with acquisition of the ability to use subsequently added features in navigation. As such, the present result is inconsistent with the automatic updating of new features into a unified spatial map, as proposed in cognitive mapping theory (O'Keefe \& Nadel, 1978). Similarly, an application of Hebbian associative learning
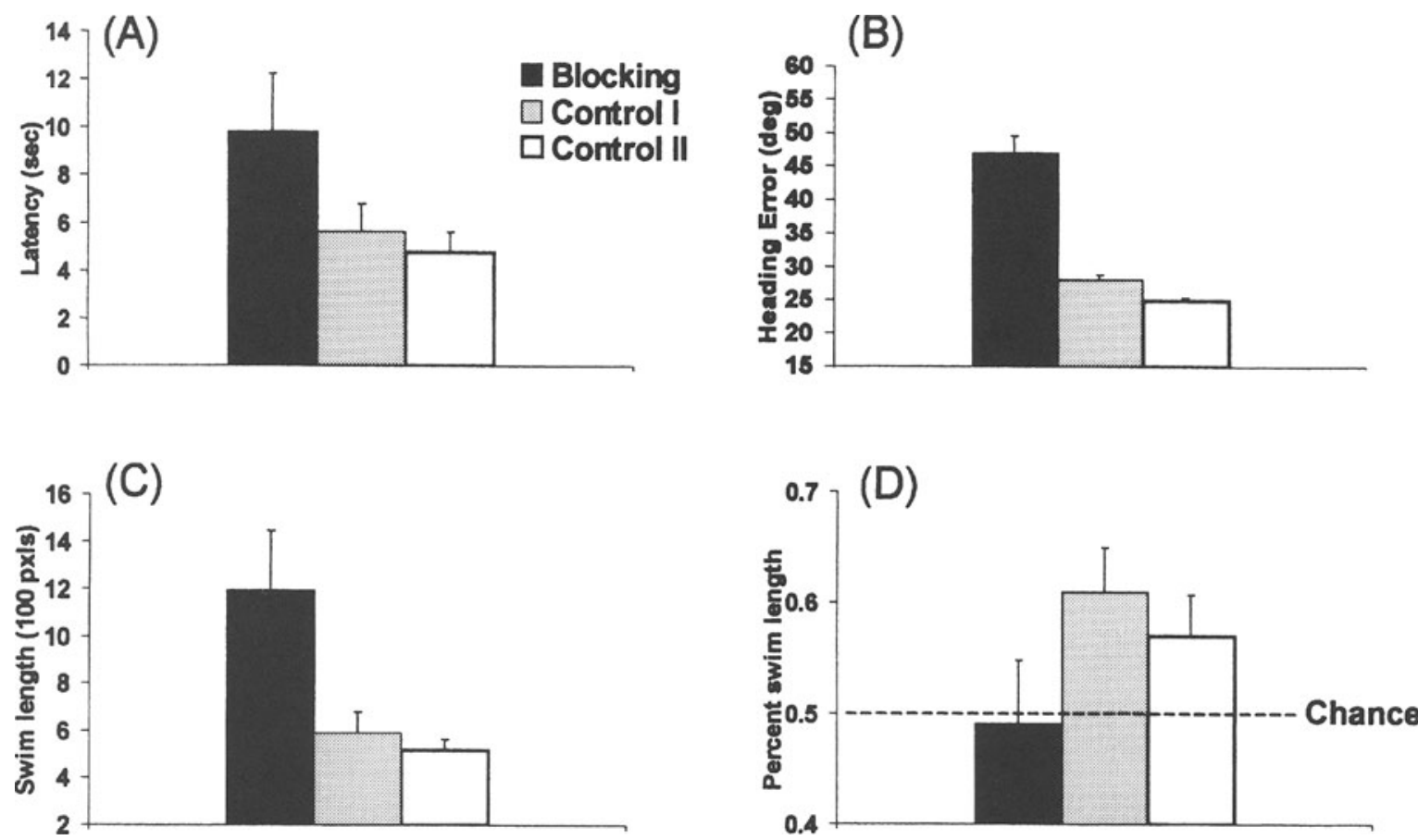

Figure 5. Phase 3 probe trial performance measures. (A) Mean latency $+1 S E M$ to reach the goal quadrant. (B) Mean heading error $+1 S E M$ calculated when a distance equal to $25 \%$ of the pool radius had been traveled from the start location. (C) Mean swim length $+1 S E M$ from start location to the goal quadrant. (D) Mean percentage of total swim length $+1 S E M$ in the goal region (a circular region centered on the platform that comprised 50\% of the total pool area). Note: Means and $S E M \mathrm{~s}$ plotted here were calculated only for place learners. 


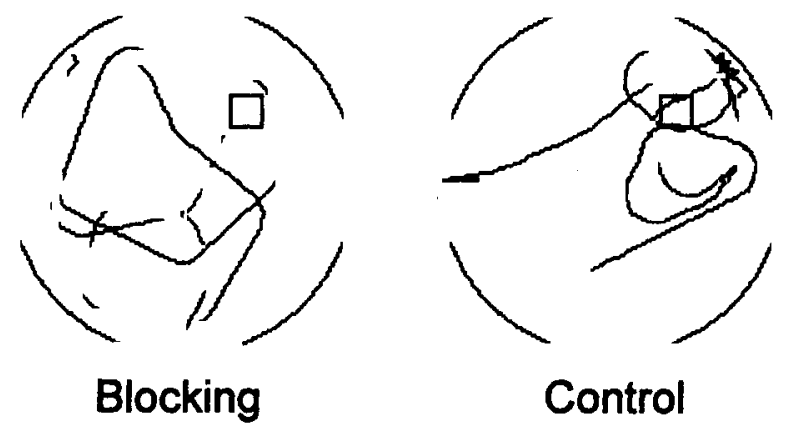

Figure 6. Representative blocking group and control group swim paths during the Phase 3 probe trial. The black square represents the platform location during training. Note: The swim paths were taken from participants classified as place learners who performed at median levels on a composite of Phase 2 training measures.

principles to spatial learning is unable to account for spatial blocking, because Hebbian learning is based solely on the contiguity of events, without respect to previously established relationships. Blocking is expected, however, if an error-correcting associative rule (see, e.g., Rescorla $\&$ Wagner, 1972) governs the acquisition of spatial associations in place learning.

A potential problem with using this outcome to decide among the various theoretical alternatives is that no measures of performance indicated increased activity, which would indicate exploration on the part of the blocking group when new cues were introduced in Phase 2. Cognitive mapping theory holds that such exploration is requisite for spontaneous updating of the cognitive map; thus, it could be argued that the participants in the blocking group never attended to or explored the new cues and, subsequently, never updated their cognitive maps. We suggest that lack of behavioral evidence, however, does not rule out exploration on the part of the blocking group. Because the new cues were interleaved with preexisting cues (see Figures 1 and 2), it is possible that the arrangement of environmental features effectively reduced the need for the participants to substantially alter their activity in order to explore the added cues. Importantly, if the new cues were noticed but not explored and, subsequently, were not capable of guiding navigation, cognitive mapping principles cannot be assumed to apply to virtual place learning in the present situation. This scenario is consistent with explanations of blocking in nonspatial domains in which redundant stimuli are noticed but do not become reliable signals for an US (Gordon, 1989, pp. 34-35; Kamin, 1969). Furthermore, Biegler and Morris (1999) report that their blocking group rats explored a novel landmark but failed to use it for goal localization. In sum, the addition of new cues does not lead to observable exploration in the present experiment, and previous work in spatial and nonspatial domains has shown that exploration, if triggered, appears insufficient for acquisition of new predictive relationships. A demonstration of unblocking (Kamin, 1969), in which a change in the predicted event at the onset of Phase 2 eliminates blocking, in place learning would support this interpretation.

A more fundamental concern not specific to the present experiment is whether virtual and real-world place learning are similar, so that generalization to and from findings in real-world navigation can follow. Virtual navigation paradigms inherently tap visual learning in the absence of inertial and proprioceptive cues from locomotion, which are used in other navigational strategies, such as path integration (Alyan \& McNaughton, 1999). Although the lack of such cues precludes the use of an entire class of strategies, place learning based on the use of visual motion information in isolation is not precluded. G. M. Martin, Harley, Smith, Hoyles, and Hynes (1998) have demonstrated intact spatial localization by disoriented rats in the MWT, suggesting that accurate vestibular information is not requisite for place navigation. Findings from virtual navigation experiments suggest that good spatial localization can occur in the complete absence of cues generated during locomotion (Astur, Ortiz, \& Sutherland, 1998). It is likely that the low proportion of participants classified as place learners $(62.2 \%)$ in part reflects this deficit, given that nearly all rats swimming in the MWT learn to take direct paths to the goal and would be classified as place learners by our criteria. In addition to inertial strategies, we rule out other nonspatial movement-based strategies as explanations of place learning in the present study. No single cue provided a direct guide to the goal location from any starting point, and four starting locations that were independent with respect to combination of distance and direction from the goal location were used. Thus, if the participants adopted a strategy of moving a certain distance in a given direction, it would be impossible to consistently take a direct path to the goal. Our contention is that the spatial localization ability reported here reflects the calculation of trajectories on the basis of the virtual topographical relationships among a configuration of distal cues and the goal, much the same as that hypothesized to be employed by rats solving the MWT. We further suggest that spatial learning in virtual environments appears to reflect realworld place learning, even though all forms of navigationrelevant information are not available in virtual navigation paradigms.

In addition to behavioral similarity, recent demonstrations of hippocampal involvement in virtual navigation suggest that real-world and virtual spatial learning involve some common neural circuitry. Maguire and colleagues have shown activation of the right hippocampus when participants navigate between familiar virtual locations (Maguire, Burgess, et al., 1998), and the right parahippocampal gyrus is activated when salient virtual cues that disambiguate locations are available in the virtual environment (Maguire, Frith, Burgess, Donnett, \& O'Keefe, 1998). In addition, Astur, Brown, et al. (1998) report failure of VMWT acquisition in patients with unilateral damage to the mesial temporal lobe, including the 
hippocampus, a finding that mirrors the devastating effects of hippocampal lesions on MWT acquisition in the rat (Morris et al., 1982; Sutherland et al., 1983).

To summarize, humans are capable of learning to navigate to a particular location in virtual space, using the configuration of a few distal cues. This ability is similar to that observed in nonhuman animals learning to place navigate in the MWT and has been shown to involve common brain systems. The degree to which humans learn to use cues as navigational guides in virtual contexts is negatively affected by the presence of previously learned goalpredictive cues in the environment. Consequently, this finding is problematic for Hebbian and cognitive mapping explanations and suggests that an error-correcting associative rule operates within the distributed placelearning neural system. However, whether an account of place learning grounded in error-correcting associative principles is justified awaits further data. Additional work is required in virtual and real-world place learning to determine whether additional phenomena, such as overshadowing and unblocking, exhibit in purely spatial tasks. If so, the operation of error-correcting rules would be further supported; however, such findings would not rule out modified cognitive mapping or Hebbian accounts as viable explanations.

\section{REFERENCES}

Alyan, S., \& McNaughton, B. L. (1999). Hippocampectomized rats are capable of homing by path integration. Behavioral Neuroscience, 113, 19-31.

Astur, R. S., Brown, N., Price, E., \& Sutherland, R. J. (1998). A characterization of processes involved in human performance in a virtual Morris water task. Society for Neuroscience Abstracts, 24, 162.

Astur, R. S, ORTIz, M. L., \& Sutherland, R. J. (1998). A characterization of performance by men and women in a virtual Morris water task: A large and reliable sex difference. Behavioural Brain Research, 93, 185-190.

BIEGler, R., \& MorRIs, R. G. M. (1999). Blocking in the spatial domain with arrays of discrete landmarks. Journal of Experimental Psychology: Animal Behavior Processes, 25, 334-351.

BuRgess, N., \& O'KeEFE, J. (1996). Neural computations underlying the firing of place cells and their role in navigation. Hippocampus, 7 , 749-762.

Gallistel, C. R. (1990). The organization of learning. Cambridge, MA: MIT Press.

GLENN, M. J., \& MUmBY, D. G. (1998). Place memory is intact in rats with perirhinal cortex lesions. Behavioral Neuroscience, 112, 1353-1365.

GoRdon, W. C. (1989). Learning and memory. Pacific Grove, CA: Brooks/Cole.

Hebs, D. O. (1949). The organization of behavior. New York: Wiley.

Hinchy, J., Lovibond, P. F., \& TERHORST, K. M. (1995). Blocking in human electrodermal conditioning. Quarterly Journal of Experimental Psychology, 48B, 2-12.

Kamin, L. (1969). Predictability, surprise, attention and conditioning. In B. A. Campbell \& R. M. Church (Eds.), Punishment and aversive behavior (pp. 279-296). New York: Appleton-Century-Crofts.

KeLSEY, J. E., \& LANDRY, B. A. (1988). Medial septal lesions disrupt spatial mapping ability in rats. Behavioral Neuroscience, 102, 289-293.

Kolb, B., Sutherland, R. J., \& WhishaW, I. Q. (1983). A comparison of the contributions of the frontal and parietal association cortex to spatial localization in rats. Behavioral Neuroscience, 97, 13-27.

MaCKINTOSH, N. J. (1975). A theory of attention: Variations in the as- sociability of stimuli with reinforcement. Psychological Review, 82, 276-298.

Mackintosh, N. J. (1997). Has the wheel turned full circle? Fifty years of learning theory, 1946-1996. Quarterly Journal of Experimental Psychology, 50A, 879-898.

Maguire, E. A., Burgess, N., Donnett, J. G., Frackowiak, R. S. J., FrITH, C. D., \& O'KeEFE, J. (1998). Knowing where and getting there: A human navigation network. Science, 280, 921-924.

Maguire, E. A., Frith, C. D., Burgess, N., Donnett, J. G., \& O'KeEFE, J. (1998). Knowing where things are: Parahippocampal involvement in encoding object locations in virtual large-scale space. Journal of Cognitive Neuroscience, 10, 61-76.

Martin, G. M., Harley, C. W., Smith, A. R., Hoyles, E. S., \& Hynes, C. A. (1998). Spatial disorientation blocks reliable goal location on a plus-maze but does not prevent goal location in the Morris maze. Journal of Experimental Psychology: Animal Behavior Processes, 23, 183-193.

Martin, I., \& Levey, A. B. (1991). Blocking observed in human eyelid conditioning. Quarterly Journal of Experimental Psychology, 43B, 233-256.

MCNaughton, B. L., \& Morris, R. G. M. (1987). Hippocampal synaptic enhancement and information storage within a distributed memory system. Trends in Neurosciences, 10, 408-415.

MorRIs, R. G. M. (1981). Spatial localisation does not require the presence of local cues. Learning \& Motivation, 12, 239-260.

MORRIS, R. G. M., \& FREY, U. (1997). Hippocampal synaptic plasticity: Role in spatial learning or the automatic recording of attended experience? Philosophical Transactions of the Royal Society of London: Series $B, 352,1489-1503$.

Morris, R. G. M., Garkud, P., Rawlins, J. N. P., \& O'KeEfe, J. (1982). Place navigation impaired in rats with hippocampal-lesions. Nature, 297, 681-683.

Moser, E. I., Krobert, K. A., Moser, M. B., \& Morris, R. G. M. (1998). Impaired spatial-learning after saturation of long-term potentiation. Science, 281, 2038-2042.

NADEL, L. (1991). The hippocampus and space revisited. Hippocampus, $1,221-229$

NADEL, L., \& Willner, J. (1980). Context and conditioning: A place for space. Physiological Psychology, 8, 218-228.

O'KeEFE, J., \& NADEL, L. (1978). The hippocampus as a cognitive map. London: Oxford University Press.

Pearce, J. M., \& Hall, G. (1980). A model for Pavlovian learning: Variations in the effectiveness of conditioned but not of unconditioned stimuli. Psychological Review, 87, 532-552.

Prados, J., \& Trobalon, J. B. (1998). Locating an invisible goal in a water maze requires at least two landmarks. Psychobiology, 26, 42-48.

Rescorla, R., \& WAGNER, A. (1972). A theory of Pavlovian conditioning: Variations in the effectiveness of reinforcement and nonreinforcement. In A. H. Black \& W. F. Prokasky (Eds.), Classical conditioning II: Current research and theory (pp. 64-99). New York: Appleton-Century-Crofts.

Rodrigo, T., Chamizo, V. D., McLaren, I. P. L., \& Mackintosh, N. J. (1997). Blocking in the spatial domain. Journal of Experimental Psychology: Animal Behavior Processes, 23, 110-118.

Rudy, J. W., \& SutheRLand, R. J. (1995). Configural association theory and the hippocampal formation: An appraisal and reconfiguration. Hippocampus, 5, 375-389.

Schenk, F., \& Morris, R. G. M. (1985). Dissociation between components of spatial memory in rats after recovery from the effects of retrohippocampal lesions. Experimental Brain Research, 58, 11-28.

SuTherland, R. J., KolB, B., \& Whishaw, I. Q. (1982). Spatial mapping: Definitive disruption by hippocampal or frontal cortical damage in the rat. Neuroscience Letters, 31, 271-276.

Sutherland, R. J., \& Rodriguez, A. J. (1989). The role of the fornix/fimbria and some related subcortical structures in place learning and memory. Behavioural Brain Research, 32, 265-277.

Sutherland, R. J., \& Rudy, J. W. (1989). Configural association theory: The role of the hippocampal formation in learning, memory, and amnesia. Psychobiology, 17, 129-144. 
Sutherland, R. J., Whishaw, I. Q., \& KolB, B. (1983). A behavioralanalysis of spatial localization following electrolytic, kainite-induced or colchicine-induced damage to the hippocampal formation in the rat. Behavioural Brain Research, 7, 133-153.

Sutherland, R. J., Whishaw, I. Q., \& Kolb, B. (1988). Contribution of cingulate cortex to two forms of spatial learning and memory. Journal of Neuroscience, 8, 1863-1872.

Tolman, E. C. (1948). Cognitive maps in rats and men. Psychological Review, 55, 189-209.

Weisend, M. P., Klein, R. L., Hoesing, J. M., Astur, R. S., Koerner, A., McDonald, R. J., Geving, T., Peinado, J., Biela, J., MCWhorter, J., WeEmS, M., SChlegelmilch, J., Yeo, R., \& SUTHERLAND, R. J. (1995). Morris water task: Which cues define locations? Society for Neuroscience Abstracts, 21, 1939.
Whishaw, I. Q., Mittleman, G., Bunch, S. T., \& Dunnett, S. B. (1987). Impairments in the acquisition, retention and selection of spatial navigation strategies after medial caudate-putamen lesions in rats. Behavioural Brain Research, 24, 125-138.

WIIG, K. A., \& BILKEY, D. K. (1994a). The effects of perirhinal cortical lesions on spatial reference memory in the rat. Behavioural Brain Research, 63, 101-109.

WIIG, K. A., \& BILKEY, D. K. (1994b). Subtotal perirhinal cortex lesions increase exploratory behavior in the rat without producing deficits in the Morris water maze. Psychobiology, 22, 195-202.

(Manuscript received May 4, 1999; revision accepted for publication August 2, 1999.) 